\title{
Pulmonary Embolism in a COVID-19-Positive Primigravida After Caesarean Section Despite Prophylaxis
}

\author{
Yash Kripalani ${ }^{1}$, Lipeeka Parulekar ${ }^{2}$ \\ ${ }^{1}$ Department of Critical Care Medicine, Holy Family Hospital Bandra, Mumbai, India \\ ${ }^{2}$ Department of General Internal Medicine, Holy Family Hospital Bandra, Mumbai, India
}

Received: 01/06/2021

Accepted: 09/06/2021

Published: $16 / 07 / 2021$

How to cite this article: Kripalani Y, Parulekar L. Pulmonary embolism in a COVID-19-positive primagravida: after caesarean section despite prophylaxis. EJCRIM 2021;8: doi:10.12890/2021_002684.

Conflicts of Interests: The authors declare there are no competing interests.

This article is licensed under a Commons Attribution Non-Commercial 4.0 License

\section{ABSTRACT}

The prevalence of venous thromboembolism (VTE) in COVID-19 patients is higher than in non-COVID-19 patients. Since the beginning of the pandemic, deep vein thrombosis, myocardial infarction, ischaemic stroke and pulmonary embolism (PE) have been reported in patients with COVID-19. D-dimer levels are now routinely measured in hospitalized patients so that prophylaxis can be initiated. However, a standardized protocol for prophylaxis has yet to be developed for pregnant women with COVID-19, who have an increased risk of VTE. We describe the case of a young primigravida woman with a positive COVID RT-PCR test who developed PE despite receiving adequate prophylaxis.

\section{LEARNING POINTS}

- $\quad$ COVID-19 patients may develop venous thromboembolism (VTE) and so adequate prophylaxis should be provided.

- VTE may still develop despite adequate prophylaxis, especially in patients at high risk.

- A standardized prophylactic protocol to prevent VTE in pregnant women should be developed to reduce mortality before and after caesarean section.

\section{KEYWORDS}

Pulmonary embolism, COVID-19, primigravida, pregnancy, caesarean section

\section{INTRODUCTION}

The prevalence of venous thromboembolism (VTE) in COVID-19 patients is higher than in other ICU patients. A significant increase in D-dimer levels indicates activation of coagulation and fibrinolysis, and is a good indicator of a high risk of VTE. The test is very sensitive but has low specificity for the detection of an active thrombotic process. However, D-dimer can also be elevated in other conditions, such as pregnancy, postoperatively, malignancy and sepsis. The administration of subcutaneous injectable low-molecular-weight heparin (LMWH), such as enoxaparin once daily, is a good prophylactic method to prevent VTE. COVID-19 infection promotes a strong inflammatory response with the release of cytokines and chemokines, and cell activation. Through interactions between inflammation, complement activation and coagulation, a hypercoagulable state is generated. Pregnancy and surgery both increase the hypercoagulable state, resulting in a higher risk of developing VTE. A standardized prophylactic protocol for pregnant women with COVID-19 has not yet been developed but is urgently needed. 


\section{CASE DESCRIPTION}

A 29-year-old primigravida woman 38.1 weeks pregnant by date and 36.6 weeks by ultrasound, presented with a 7-day history of fever, feeling cold and body aches. She had known hypothyroidism. She was diagnosed with COVID-19 infection with a positive RT-PCR swab. She did not complain of shortness of breath, lower limb tenderness, calf swelling or redness. Her laboratory reports showed elevated D-dimer $(1.50 \mathrm{mg} / \mathrm{l})$. She was admitted to hospital for further management. She was administered injectable enoxaparin (Clexane) subcutaneously 60 $\mathrm{mg}$ daily in view of the raised D-dimer level and her pregnancy. She was scheduled for an elective lower segment caesarean section (LSCS). Obstetric examination determined a floating head with a Bishop score of 5. Injectable enoxaparin was withheld the day before the LSCS procedure. The LSCS was uneventful and a healthy baby weighing $3.5 \mathrm{~kg}$ was born. The patient was then moved to the ICU for recovery and monitoring. Deep vein thrombosis (DVT) pumps were used as prophylaxis to prevent VTE as injectable enoxaparin could not be started immediately. Enoxaparin was restarted the next day at the same dosage as the patient was at risk of VTE. On the second day after surgery, she complained of difficulty breathing with chest heaviness. She received an injection of remdesivir as an antiviral medication since she was positive for COVID-19.

We began work-up for pulmonary embolism (PE) immediately. Her subsequent post-operative laboratory results are given in Table 1. The D-dimer levels were still elevated (1.80 mg/l). 2D Echocardiography did not show any signs of pulmonary hypertension or dilated right chambers of the heart. An ultrasound Doppler scan was conducted but did not reveal any signs of DVT in the lower limbs. A CT pulmonary angiogram of the chest was performed and showed signs of a hypodense filling defect, suggestive of pulmonary thromboembolism in the anterior basal and lateral basal segmental and subsegmental branches of the right lower lobar pulmonary artery (Fig. 1). The pulmonary trunk, right and left main pulmonary arteries and the rest of the segmental branches showed normal opacification and calibre. Injectable enoxaparin was increased to $60 \mathrm{mg}$ twice daily, which was later bridged to oral warfarin and the International Normalized Ratio (INR) was monitored. Our patient stabilized and was eventually discharged.
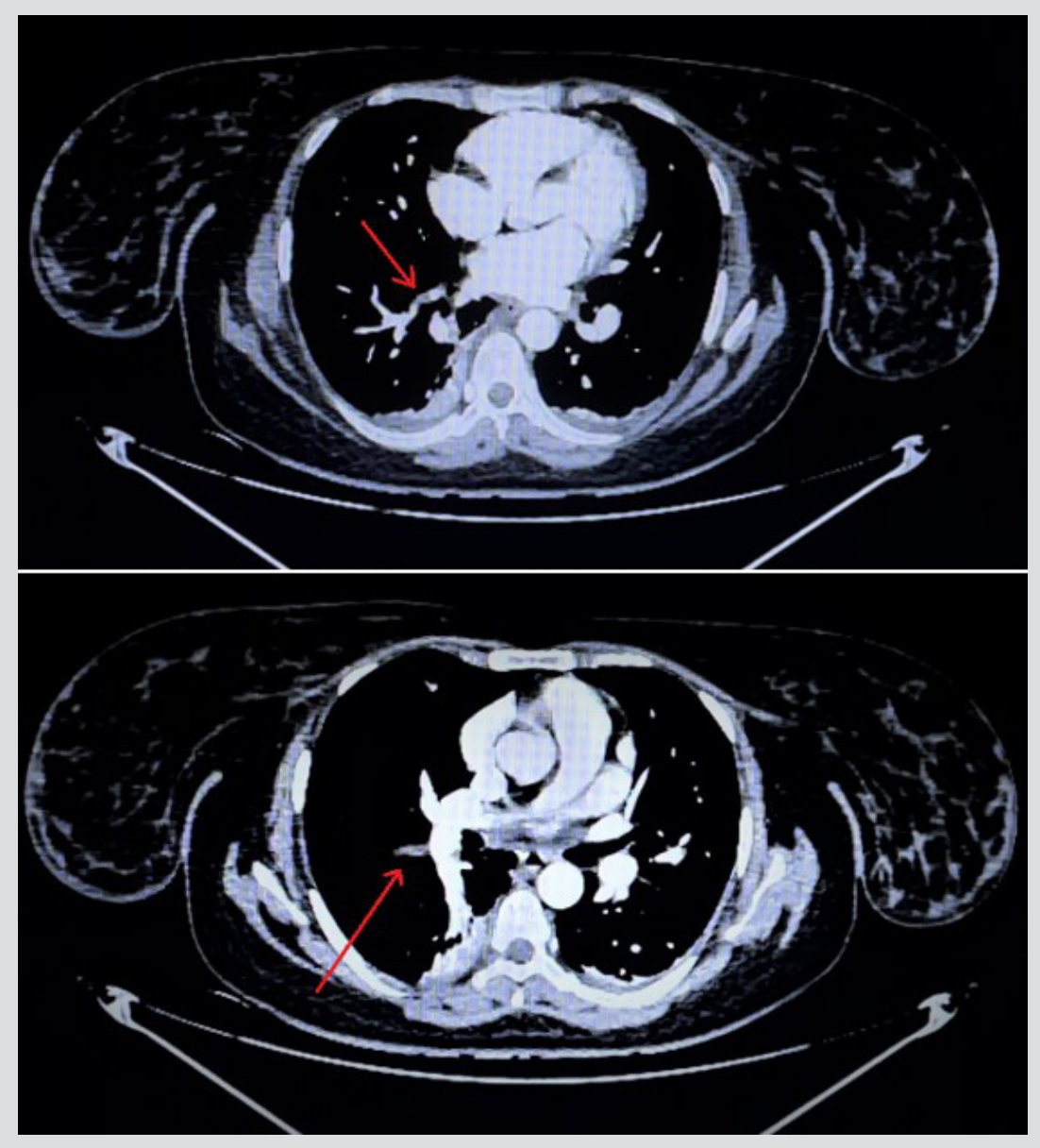

Figure 1. CT pulmonary angiogram of the chest, axial cut view, showing a hypodense filling defect, suggestive of pulmonary thromboembolism 


\begin{tabular}{|l|l|}
\hline Laboratory investigation & Value \\
\hline D-dimer & $1.80 \mathrm{mg} / \mathrm{l}$ \\
\hline Total leukocyte count & $18,180 / \mathrm{mm}^{3}$ \\
\hline Neutrophils & $82 \%$ \\
\hline Lymphocytes & $12 \%$ \\
\hline Neutrophil-to-lymphocyte ratio & 6.8 \\
\hline Platelets & $208 \times 10^{3} / \mu l$ \\
\hline Anti-phospholipid antibody & Negative \\
\hline
\end{tabular}

Table 1. Post-operative laboratory results

Despite all prophylactic measures, our patient developed PE. The development of a standardized VTE prophylactic protocol for pregnant women with COVID-19 is urgently required as they are at risk of VTE, which can be fatal.

\section{DISCUSSION}

Since the possibility of VTE increases with COVID-19 severity, many patients with VTE are at risk of multiorgan failure. Risk factors for VTE in COVID-19 patients include older age, obesity, immobilization, smoking and comorbidities, such as prior history of VTE, chronic kidney disease, malignant tumour and heart/respiratory failure. A reduced lymphocyte count is common in patients with COVID-19, which increases the risk of thrombosis, so elderly patients with underlying disease are more prone to immune dysfunction due to weakened immunity and so have a higher risk of VTE. In addition, platelet count, prothrombin time (PT), activated partial thromboplastin time (aPTT), antithrombin, fibrinogen, fibrinogen degradation product (FDP), and other related coagulation parameters can be measured to indicate thrombosis risk. Several studies have found that prolonged PT in COVID-19 patients may be related to the severity of the disease and mortality.

In COVID-19, interaction between thrombosis and inflammation results in a hypercoagulable state through mechanisms unique to severe acute respiratory syndrome coronavirus 2 (SARS-CoV-2). First, SARS-CoV-2 enters the alveolar epithelium via the angiotensin converting enzyme 2 (ACE2) receptor, causing excessive release of inflammatory cytokines (such as interleukin (IL)-6 and tumour necrosis factor) and chemokines (IL-8, CXCL8, CCL2 and CCL3). This results in activation of epithelial cells, monocytes and neutrophils. Alternatively, endothelial cells can be directly infected through the ACE2 receptor, leading to endothelial activation and dysfunction, thereby triggering a coagulation cascade that generates thrombin and fibrin clots. The activation of platelets and the protease-activated receptor signalling pathway during the process further stimulates inflammation causing a higher pro-inflammatory state which leads to coagulation lesions. Studies have reported that a few patients with severe COVID-19 infection express antiphospholipid antibodies, which may increase the risk of thrombosis. The inflammatory response and cytokine storm can raise the risk of VTE. Pregnancy and surgery further increased the risk of VTE in our patient ${ }^{[1-3]}$.

Routine screening of D-dimers and subcutaneous LMWH injection are the gold standard treatments to prevent VTE. 2D Echocardiography of the heart is also important for screening the heart for PE, which is a life-threatening event. The vast majority of studies on prophylaxis against VTE in COVID-19 have used LMWH. Compared with unfractionated heparin (UFH), it has the advantages of once-daily injection with more predictable pharmacokinetics, less binding to plasma proteins, particularly to acute-phase reactants, and a lower risk for heparininduced thrombocytopenia. Anticoagulation treatment is the mainstay of therapy for every patient with acute VTE. Especially in the case of PE, current guidelines emphasize that anticoagulation should be immediately initiated upon clinical suspicion in patients with high or intermediate clinical (pretest) probability while awaiting the results of diagnostic imaging tests. Subcutaneous, weight-adjusted therapeuticdose LMWH is the first choice, while subcutaneous fondaparinux or intravenous infusion of UFH may be used as an alternative. Novel oral anticoagulants (NOACs; apixaban, rivaroxaban, edoxaban or dabigatran) are preferred over vitamin K antagonists (VKAs) for chronic oral treatment and secondary prophylaxis. For admitted patients receiving experimental antiviral treatments, the interaction between some of 
the experimental treatments and NOACs may result in very high plasma NOAC levels. DVT compression stockings and calf pumps applied to both lower limbs further aid in prophylactic management for DVT and reduce the risk of VTE significantly ${ }^{[1,2,4]}$.

Pregnant women have a five-fold higher risk of VTE than non-pregnant women of the same age. Women generally develop postpartum rather than ante-partum VTE. Women with congenital abnormalities or persistent presence of antiphospholipid antibodies have an increased risk of VTE during pregnancy and the puerperium. Clinical diagnosis of DVT is generally difficult and is further hampered during pregnancy since dyspnoea, tachypnoea, and swelling and discomfort in the legs are common. Therefore, in addition to sonography, routine diagnostic procedures should be performed if clinically necessary. Patients who have hereditary antithrombin deficiency, antiphospholipid antibodies, a combined abnormality or a history of a severe thrombotic event (PE, extended DVT) should be advised to use prophylactic heparin during pregnancy, starting during the first trimester. Postpartum prophylaxis should be given to all women with an increased risk of VTE ${ }^{[5]}$.

Our patient did not have any sign initially other than a raised D-dimer that put her at a risk of VTE, thus prophylaxis was given. Despite the provision of adequate prophylaxis, our patient developed VTE on the second post-operative day. She was successfully managed with no further complications and was discharged on oral warfarin.

\section{CONCLUSION}

COVID-19 increases the risk of VTE. Our case demonstrates that a pregnant woman with COVID-19 and high D-dimer levels has a very high risk of developing PE after LSCS surgery. Despite the provision of adequate prophylaxis with 60 mg subcutaneous enoxaparin daily and the use of DVT pumps, our patient still developed PE. A protocol for prophylaxis against VTE with dosage recommendations in such patients needs to be developed to reduce mortality by decreasing the risk of VTE before and after procedures like LSCS. This prophylaxis protocol should be designed in such a way that it prevents complications such as post-partum haemorrhage and placental abruption as well as reducing the risk of VTE during the puerperium.

\section{REFERENCES}

1. Schulman S, Hu Y, Konstantinides S. Venous thromboembolism in COVID-19. Thromb Haemost 2020;120(12):1642-1653. doi: 10.1055/s-0040-1718532. PMID: 33099284 PMCID: PMC7869046.

2. Aryal MR, Gosain R, Donato A, Pathak R, Bhatt VR, Katel A, et al. Venous thromboembolism in COVID-19: towards an ideal approach to thromboprophylaxis, screening, and treatment. Curr Cardiol Rep 2020;22(7):52. doi: 10.1007/s11886-020-01327-9. PMID: 32529517; PMCID: PMC7288258.

3. Klok FA, Kruip MJHA, an der Meer NJM, et al. Incidence of thrombotic complications in critically ill ICU patients with COVID-19. Thromb Res 2020;191:145-147. doi: https:// doi.org/10.1016/j.thromres.2020.04.013

4. Miranda-Bacallado J, Izquierdo-Gómez MM, García-Niebla J, Jiménez JJ, Iribarren JL, Laynez-Cerdeña I, et al. Role of echocardiography in a patient with suspected acute pulmonary embolism: a case report. J Med Case Rep 2019;13(1):37. doi: 10.1186/s13256-019-1994-y. PMID: 30777120; PMCID: PMC6379930.

5. Pabinger I, Grafenhofer H. Thrombosis during pregnancy:riskfactors, diagnosis and treatment. Pathophysiol Haemost Thromb 2002;32(5-6):322-324.doi: 10.1159/000073590. PMID: 13679666. 